\title{
BRIOZOOS FENESTRADOS DE LA FORMACIÓN MONIELLO (DEVÓNICO) EN ARNAO (ASTURIAS, ESPAÑA). II: SEPTOPORIDAE, POLYPORIDAE Y ACANTHOCLADIIDAE
}

\author{
Juan Luis SUÁREZ ANDRÉS \\ Universidad de Oviedo, Departamento de Geología, Area de Paleontología. \\ Arias de Velasco s/n. 33005 Oviedo (Asturias).
}

\begin{abstract}
Suárez Andrés, J. L. 1999. Briozoos Fenestrados de la Formación Moniello (Devónico) en Arnao (Asturias, España). II: Septoporidae, Polyporidae y Acanthocladiidae.[Fenestrate Bryozoa from the Moniello Formation (Devonian) in Arnao (Asturias, Spain). II: Septoporidae, Polyporidae and Acanthocladiidae]. Revista Española de Paleontología, n" extr. Homenaje al Prof. J. Truyols, 185-193. ISSN 02 13-6937.
\end{abstract}

\begin{abstract}
The systematic study on the Fenestrida (Bryozoa) of the Moniello Formation (upper Emsian-lower Eifelian, Asturias), initiated in an earlier paper on the Fenestellidae (Suárez Andrés, 1998), is now completed. The families Septoporidae, Polyporidae and Acanthocladiidae are dealt with. Five species belonging to four genera are described: Septopora sp. A, Polypora aff. inusitata, Polypora cf. laevinodata, Ptilopora aff. bohemica and Filites sp. A. The genera Polyporella and Penniretepora wich are also present, have not been analysed because of insufficient material. Clear affinities exist with faunas from other localities in the Old World. The faunal composition and the presence of Filites in particular fit the pattern of Lower Devonian fenestrates from the Prague Basin (Bohemia). Other taxa, such as Polypora cf. laevinodata and the cryptostome ptilodictyine Intrapora, are found to be in common with eastern North America.
\end{abstract}

Keywords: Devonian, Bryozoans, Fenestrates, Septoporidae, Polyporidae, Acanthocladiidae, Asturias, Spain, paleobiogeography.

\section{RESUMEN}

Se completa el primer estudio sistemático de los briozoos Fenestrados de la Formación Moniello (Emsiense superior-Eifeliense inferior, Asturias) llevado a cabo por el autor, y cuyos resultados ya han sido expuestos parcialmente en un trabajo anterior (Suárez Andrés, 1998). El presente trabajo se centra en las familias Septoporidae, Polyporidae y Acanthocladiidae. Se han analizado cinco especies correspondientes a cuatro géneros: Septopora sp. A, Polypora aff. inusitata, Polypora cf. laevinodata, Ptilopora aff. bohemica y Filites sp. A. Se ha detectado además la presencia de Polyporella y Penniretepora, que no han sido analizados aún debido a la escasez de ejemplares hallados. La asociación de taxones indica afinidades claras con faunas de otras localidades del Dominio del Viejo Mundo. La composición de la fauna en general y la presencia del género Filites coinciden especialmente bien con el Devónico Inferior de la Cuenca de Praga, en Bohemia. Otros taxones, como Polypora cf. laevinodata y el criptostomado ptilodictyino Intrapora son comunes con el Dominio del E de Norte América.

Palabras clave: Devónico, Briozoos, Fenestrados, Septoporidae, Polyporidae, Acanthocladiidae, Asturias, España, paleobiogeografía.

\section{INTRODUCCIÓN}

A continuación se describen y figuran cinco especies de briozoos Fenestrados pertenecientes a las familias Septoporidae, Polyporidae y Ancanthocladiidae, del miembro superior de la Formación Moniello en Arnao (Asturias). En un trabajo anterior publicado en esta misma revista (Suárez Andrés, 1998), se describen los representantes de la familia Fenestellidae de la misma localidad y se hace referencia a la situación del yacimiento. Por esta razón, se remite al lector a los apartados de Introducción, Situación, Estratigrafía y Técnicas de Estudio, y a la Fig. 2 de dicho trabajo. La distribución estratigráfica de todos los taxones identificados se muestra en la Fig. 1 del presente estudio. Las abreviaturas de los caracteres morfométricos tabulados son las siguientes: ER: espesor de la rama; AR: anchura de la rama; LF: longitud de la fenéstrula; AD: 


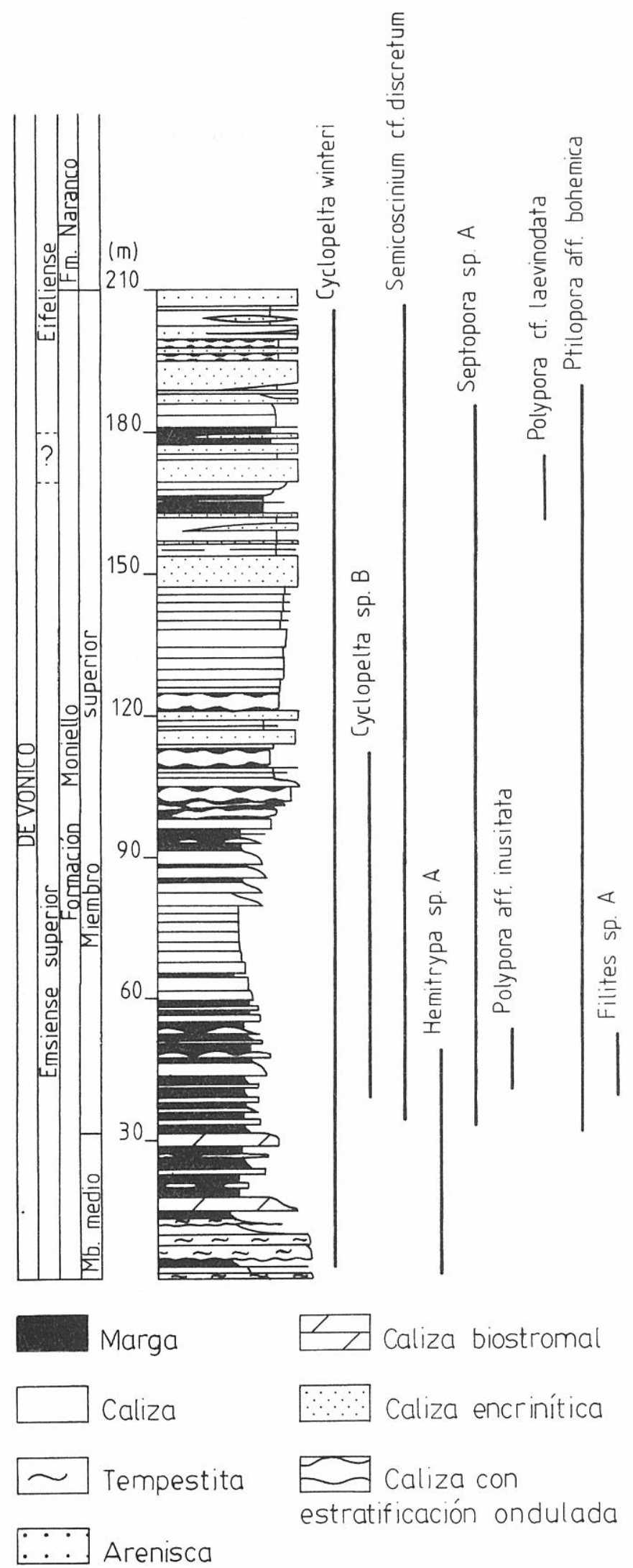

Figura 1.- Columna estratigráfica parcial de la Formación Moniello entre El Pical y la Playa de Arnao (Suárez Andrés, 1998, Fig. 1 C), con la distribución de las especies de Fenestrados identificadas. anchura de disepimento; DCR: distancia entre centros de ramas; SRL: espaciado de ramas laterales (sólo Acanthocladiidae); ALC: ángulo entre las ramas laterales y la rama principal; DAH: distancia entre centros de aberturas en una hilera; DAR: distancia entre centros de abertura a través de una rama; AA: anchura de la abertura zoecial; MAC: máxima anchura de la cámara zoecial; PC: profundidad de la cámara zoecial; LC: longitud de la cámara zoecial; ARC: ángulo agudo entre la lámina basal y la pared zoecial transversal. PC y LC se utilizan sensu Hageman (1991). La "p" y la "l" que acompañan a algunos caracteres indican que éstos corresponden a ramas principales y laterales, respectivamente. Un asterisco delante de un carácter indica que se dispone de menos de 12 medidas.

\section{SISTEMÁTICA}

FILO BRYOZOA Ehremberg, 1831

CLASE STENOLAEMATA Borg, 1926

ORDEN FENESTRIDA Elias y Condra, 1957

Familia Septoporidae Morozova, 1962

Septopora Prout, 1859

Especie tipo: Septopora cestriensis Prout, 1859.

\section{Diagnosis}

Zoario reticulado formado por numerosas ramas principales conectadas por ramas laterales. Ramas principales producidas por prolongación de ramas laterales o por bifurcación de otras ramas principales. Ramas laterales unidas con las ramas laterales de ramas principales adyacentes, formando un ángulo cuyo vértice apunta en sentido distal. Dos hileras de aberturas en ramas principales y laterales. Reverso comúnmente estriado, ciclozoecios dispersos comúnmente presentes. (Basado en Nickles y Bassler, 1900).

\section{Septopora sp. A \\ Lám. I, figs. 1-3}

\section{Descripción}

Zoario en forma de abanico; ramas principales rectas o ligeramente sinuosas; ramas laterales cortas, unidas con las de las ramas principales vecinas formando un ángulo generalmente agudo, próximo a los $90^{\circ}$, cuyo vértice apunta en sentido distal. Cuando una rama lateral no se termina contra otra, se dispone paralela a las principales a medida que crece, transformándose en una nueva rama principal (Lám. I, fig. 3). La nueva rama principal no genera ramas laterales en los primeros estadios de su desarrollo, y las ramas laterales de las ramas principales vecinas se unen directamente a ella formando un ángulo agudo. Dos hileras de zoecios en ramas principales y laterales separadas por una quilla baja. Ramas principales en ocasiones levemente sinuosas, coincidiendo las convexidades con el nacimiento de ramas laterales (Suárez Andrés, 1997; lám. 4 fig. 1). Espesamiento del reverso de las ramas con esqueleto laminar, más pronunciado en las principales que en las 
laterales. Microestiletes presentes en el reverso (Lám. I, figs. 1, 2). Zoecios en dos hileras alternas separadas por una pared axial recta. Cámaras rectangulares o con forma de romboide, alargadas y poco profundas. Parte distal rampante, desembocando en un tubo distal escasamente divergente de la pared axial, inclinado en sentido distal (Lám. I, fig. 2). Paredes zoeciales laterales planas en sección tangencial, ligeramente infladas en sección transversal. Lámina basal cóncava, casi ojival en sección transversal, con un número reducido de crestas longitudinales en el reverso (Lám. I, fig. 1). Los valores de los caracteres morfométricos de Septopora sp. A se encuentran en la Tabla 1.

\section{Discusión}

Aunque es frecuente la presencia de ciclozoecios, en mayor o menor medida, en las especies de Septopora, no se han podido reconocer con total seguridad en el material estudiado; ocasionalmente aparecen en el reverso de las ramas pequeños tubos de sección circular que podrían ser bien perforaciones o bien estos pequeños polimorfos.

\section{Familia Polyporidae Vine, 1880 Polypora McCoy, 1844}

\section{Especie tipo: Polypora dendroides McCoy, 1844.}

\section{Diagnosis}

Zoario reticulado con fenéstrulas medianas o grandes, ramas rectas o ligeramente sinuosas conectadas a intervalos regulares por disepimentos estériles. Cuatro hileras de zoecios por rama, presentando cinco o seis antes de una bifurcación y dos o tres después. Cámaras zoeciales hexagonales o romboidales. Zoecios tubulares con hemisepto inferior poco desarrollado. Aberturas zoeciales circulares. No existen crestas separando hileras vecinas de zoecios (Morozova y Lisitsyn, 1996).

\section{Polypora aff. inusitata McKinney y Kříž, 1986 Lám. I, figs. 4-6; Lám. II, fig. 1}

\section{Descripción}

Zoario posiblemente con forma de abanico (sólo se han encontrado fragmentos, en general planos). Ramas rectas con cuatro hileras de zoecios (cinco a seis en las bifurcaciones y tres a cuatro tras las bifurcaciones). Microestiletes en el anverso. Marcado ensanchamiento proximal y estrechamiento distal a las bifurcaciones. Disepimentos estrechos con finas estrías longitudinales, separados entre sí por una distancia variable, conformando fenéstrulas muy alargadas, raramente elípticas (Lám. I, fig. 6). Esqueleto laminar grueso en el reverso de las ramas, concentrado en la parte media más que en los flancos, produciendo una sección transversal con forma subtriangular. Reverso con abundantes microestiletes de muy pequeño tamaño. Lámina basal cóncava con numerosas estrías longitudinales (Lám. II, fig. 1). Cámaras zoeciales de forma rómbica irregular en sección tangencial profunda, ovalada o elíptica en secciones más someras (Lám. I, fig. 5). Tubos distales inclinados en el sentido de crecimiento

$\begin{array}{lcccc} & \text { media } & \text { desviación } & \text { rango } & \text { cf. varianza } \\ \text { *ERp } & 0,825 & 0,0150 & 0,81-0,84 & 1,81 \\ \text { *ERl } & 0,625 & 0,0290 & 0,58-0,65 & 4,59 \\ \text { ARp } & 0,559 & 0,0520 & 0,53-0,68 & 9,36 \\ \text { ARl } & 0,434 & 0,0306 & 0,398-0,466 & 7,06 \\ \text { DCRl } & 0,751 & 0,0694 & 0,665-0,893 & 9,24 \\ \text { ALC } & 48,92 & 4,9742 & 42-57 & 10,17 \\ \text { DAHp } & 0,260 & 0,0155 & 0,233-0,293 & 5,95 \\ \text { DAHl } & 0,285 & 0,0201 & 0,266-0,31 & 6,81 \\ \text { DARp } & 0,285 & 0,0228 & 0,26-0,32 & 7,99 \\ \text { DARl } & 0,254 & 0,0153 & 0,23-0,28 & 6,01 \\ \text { *AAp } & 0,125 & 0,0126 & 0,11-0,15 & 10,07 \\ \text { *MACp } & 0,130 & 0,0041 & 0,125-0,135 & 3,14\end{array}$

Tabla 1. Medidas de Septopora sp. A. Las abreviaturas de los caracteres utilizados se explican en la Introducción. Las medias y rangos están expresados en mm, excepto para el carácter ALC, en grados.

zoarial, verticales en zoecios de filas centrales e inclinados hacia las fenéstrulas en los de filas laterales (Lám. I, fig. 4; Lám. II, fig. 1). Aberturas petaloides con peristoma poco prominente. Los valores de los caracteres morfométricos de Polypora aff. inusitata se encuentran en la Tabla 2.

\section{Discusión}

Esta especie es muy similar a Polypora inusitata en la mayoría de los caracteres zoariales y zoeciales, excepto en la anchura de las cámaras, ligeramente inferior en aquella. La forma petaloide de las aberturas en nuestros ejemplares tampoco se menciona en la descripción de $P$. inusitata.

Polypora inusitata aparece en la Cuenca de Praga desde el Praguiense superior al Zlichoviense (Emsiense inferior); la distribución de Polypora aff. inusitata se muestra en la Figura 1.

\section{Polypora cf. laevinodata (Hall, 1881) Lám. I, fig. 7}

\section{Descripción}

Zoario compuesto por ramas sinuosas, unidas por disepimentos cortos y deprimidos situados en las convexidades de las ramas. Once o doce ramas en $10 \mathrm{~mm}$. Siete u ocho fenéstrulas elípticas en $10 \mathrm{~mm}$. Cuatro hileras de zoecios en cada rama, aumentando a seis antes y disminuyendo a tres inmediatamente después de las bifurcaciones. Aberturas subcirculares con peristoma grueso (Lám. 1, fig. 7), dispuestas según un patrón rómbico; las situadas en las uniones de ramas con disepimentos están desplazadas hacia el disepimento. Los valores de los caracteres morfométricos de Polypora cf. laevinodata se encuentran en la Tabla 3.

\section{Discusión}

Polypora cf. laevinodata es muy similar a $P$. laevinodata (Hall) en cuanto a anchura de la rama, número de ramas en $10 \mathrm{~mm}$ (carácter comparable a DCR), número de aberturas en $5 \mathrm{~mm}$ (carácter comparable a DAH) y 


\begin{tabular}{|c|c|c|c|c|c|c|c|c|c|}
\hline & media & desviación & rango & cf. varianza & & media & desviación & rango & cf. varianza \\
\hline ER & 0,596 & 0,0398 & $0,52-0,67$ & 6,67 & $\mathrm{AR}$ & 0,5427 & 0,0548 & $0,45-0,64$ & 10,05 \\
\hline AR & 0,614 & 0,0884 & $0,53-0,74$ & 14,40 & LF & 0,9810 & 0,0788 & $0,88-1,16$ & 8,03 \\
\hline LF & 1,662 & 0,2743 & $1,25-2,23$ & 16,91 & $* A D$ & 0,4312 & 0,1604 & $0,27-0,69$ & 37,20 \\
\hline $\mathrm{AD}$ & 0,430 & 0,0679 & $0,28-0,53$ & 15,78 & DCR & 0,9290 & 0,0962 & $0,77-1,05$ & 10,35 \\
\hline DCR & 1,214 & 0,1598 & $0,95-1,55$ & 13,16 & DAH & 0,2575 & 0,0128 & $0,24-0,286$ & 4,98 \\
\hline DAH & 0,312 & 0,0188 & $0,28-0,34$ & 6,01 & DAR & 0,2174 & 0,0974 & $0,20-0,244$ & 8,00 \\
\hline DAR & 0,251 & 0,0125 & $0,24-0,28$ & 5,00 & $\mathrm{AA}$ & 0,1170 & 0,0067 & $0,11-0,127$ & 5,72 \\
\hline
\end{tabular}

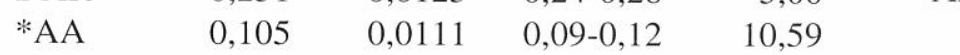

$\begin{array}{lcccr}\text { MAC } & 0,167 & 0,0098 & 0,16-0,18 & 5,85 \\ \text { *PC } & 0,160 & 0,0089 & 0,15-0,175 & 5,54 \\ \text { LC } & 0,300 & 0,0346 & 0,28-0,345 & 11,52 \\ \text { *ARC } & 72 & 2,6077 & 68-75 & 3,62\end{array}$

Tabla 3. Medidas de Polypora cf. laevinodata (Hall, 1881). Las abreviaturas de los caracteres utilizados se explican en la Introducción. Las medias y rangos están expresados en mm.

Tabla 2. Medidas de Polypora aff. inusitata McKinney y Krúiż, 1986. Las abreviaturas de los caracteres utilizados se explican en la Introducción. Las medias y rangos están expresados en mm, excepto para el carácter ARC, en grados.

número de fenéstrulas en $10 \mathrm{~mm}$. La anchura de fenéstrula medida por Stratton y Horowitz (1977a) es equivalente al carácter denominado aquí DCR, y presenta valores muy parecidos. Se desconocen las características internas tanto de la especie americana como de los ejemplares asturianos por lo que se ha preferido mantener la especie como Polypora cf. laevinodata.

Polypora laevinodata fue definida en el Grupo Hamilton (Devónico Medio) de Nueva York y posteriormente citada por Stratton y Horowitz (1977b) en el Eifeliense superior de Indiana.

\section{Familia Acanthocladiidae Zittel, 1880 Ptilopora McCoy, 1844}

Especie tipo: Ptilopora pluma McCoy, 1844.

\section{Diagnosis}

Zoario pinnado en forma de abanico, compuesto por una rama principal robusta, fuertemente espesada por esqueleto laminar, que presenta a cada lado ramas laterales más delgadas, equidistantes, conectadas por disepimentos. Dos hileras de zoecios en ramas principales y laterales. Anverso de las ramas más o menos aplanado, con una quilla por lo general provista de nodos.

\section{Ptilopora aff. bohemica Prantl, 1928 Lám. II, figs. 2-5}

\section{Descripción}

Zoario pinnado con varias ramas principales rectas; ramas laterales largas, con un ángulo de divergencia respecto a la rama principal disminuyendo a lo largo del crecimiento, conectadas por disepimentos. Superficie del zoario con estrías longitudinales. Ramas principales y laterales con dos hileras de zoecios separadas por una quilla poco prominente provista de nodos uniseriados (Lám. II, fig. 5). Algunas ramas laterales, cuando son muy numerosas o las ramas principales están muy próximas, se terminan unas contra otras o contra ramas principales vecinas. Pueden formarse ramas de tercer orden en los flancos de ramas laterales bien desarrolladas. Esqueleto laminar espeso en las ramas principales (Lám. II, fig. 4), especialmente en el reverso; ramas laterales menos reforzadas. Disepimentos irregularmente espaciados, delimitando fenéstrulas de dimensiones y morfología variables. Comúnmente son subrectangulares a elípticas, más o menos alargadas en función del espaciado de disepimentos y del espesor del esqueleto laminar. En zonas con elevada densidad de ramas, la morfología es irregular y el tamaño muy variable. Zoecios en dos hileras alternas separadas por una pared media sinuosa en sección tangencial profunda, volviéndose progresivamente más recta hacia el anverso, hasta terminar en la quilla (Lám. II, fig. 5). Cámaras zoeciales pentagonales alargadas en sección tangencial profunda, con las paredes laterales planas o ligeramente infladas; pentagonales o triangulares en ramas laterales. Tubo distal largo, inclinado

\section{Lámina I}

1-3 Septopora sp. A. 1: sección transversal de una rama principal de la que nace una lateral (derecha); se observan numerosos microestiletes. PIC-29s-7, x79. 2: sección longitudinal del mismo ejemplar, mostrando la morfología de los zoecios; x79. 3: sección tangencial superficial, profundizando hacia la parte superior de la fotografía. Se observa un posible ciclozoecio en la rama principal (flecha); PIC-30-5, x26.

4-6 Polypora aff. inusitata. McKinney y Kříž, 1986. 4: sección longitudinal mostrando las cámaras zoeciales y

la abertura de una cámara lateral abriéndose hacia la fenéstrula. PIC-29-2, x28. 5: sección tangencial del mismo ejemplar; se aprecian cuatro hileras de zoecios y su morfología; x28. 6: exterior del anverso, se observa la variación en la anchura de las ramas y el número de hileras de zoecios antes y después de las bifurcaciones. PIC-35-1, x8.

7 Polypora cf. laevinodata (Hall, 1881). Anverso de un fragmento de zoario mostrando la sinuosidad de las ramas y el grosor de los peristomas. PIC 61-6, x9. 

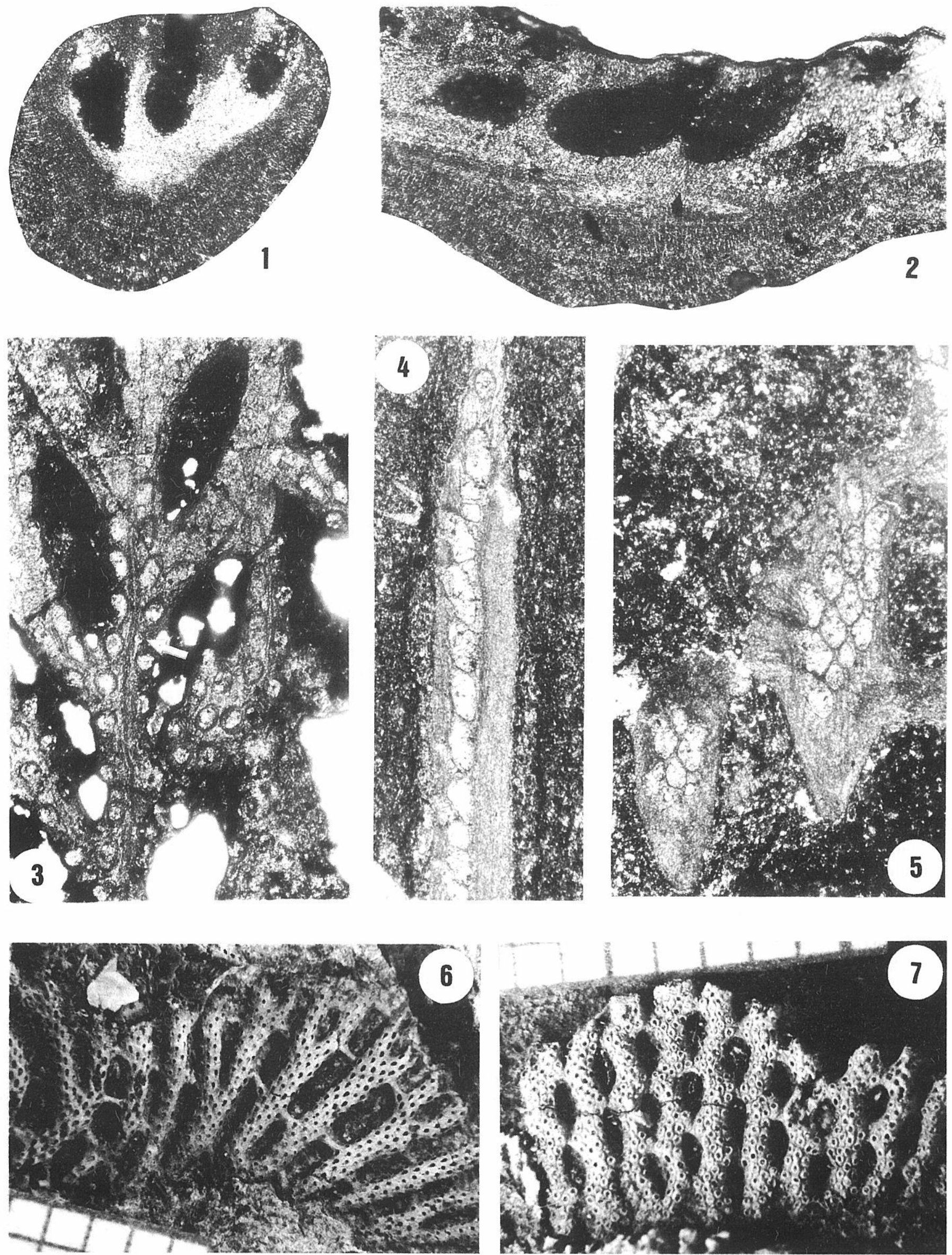

Revista Española de Paleontología, nº extr. Homenaje al Prof. J. Truyols, 1999. 


$\begin{array}{lcccc} & \text { media } & \text { desviación } & \text { rango } & \text { cf. varianza } \\ \text { ERp } & 0,5775 & 0,0549 & 0,50-0,65 & 9,51 \\ \text { ERl } & 0,2658 & 0,0752 & 0,16-0,40 & 28,29 \\ \text { ARp } & 0,5158 & 0,0585 & 0,43-0,60 & 11,34 \\ \text { ARl } & 0,2825 & 0,0283 & 0,25-0,35 & 10,02 \\ \text { LF } & 0,4042 & 0,1376 & 0,12-0,70 & 34,05 \\ \text { AD } & 0,3383 & 0,0920 & 0,20-0,50 & 27,19 \\ \text { DCRl } & 0,5450 & 0,1613 & 0,35-0,85 & 29,60 \\ \text { SRL } & 0,2700 & 0,1600 & 0,10-0,55 & 59,26 \\ \text { ALC } & 26,75 & 6,4566 & 17-42 & 24,14 \\ \text { DAHp } & 0,2450 & 0,0176 & 0,21-0,28 & 7,16 \\ \text { *DAHl } & 0,234 & 0,0174 & 0,22-0,26 & 7,45 \\ \text { DARp } & 0,2688 & 0,0204 & 0,23-0,30 & 7,60 \\ \text { *DARl } & 0,2128 & 0,0183 & 0,20-0,25 & 8,59 \\ \text { AAp } & 0,1125 & 0,0080 & 0,10-0,12 & 7,14 \\ \text { MACp } & 0,1183 & 0,0077 & 0,11-0,13 & 6,53 \\ \text { PCp } & 0,1395 & 0,0083 & 0,125-0,15 & 5,98 \\ \text { LCp } & 0,2246 & 0,0116 & 0,21-0,245 & 5,18 \\ \text { *ARCp } & 66,6 & 0,9428 & 66-68 & 1,41\end{array}$

Tabla 4. Medidas de Ptilopora aff. bohemica Prantl, 1928. Las abreviaturas de los caracteres utilizados se explican en la Introducción. Las medias y rangos están expresados en mm, excepto los caracteres ALC y ARC, en grados.

distalmente y hacia la fenéstrula (Lám. II, figs. 2, 3). Paredes zoeciales laterales infladas en sección transversal; esqueleto granular grueso con numerosas proyecciones hacia el esqueleto laminar y estrías en el reverso. Los valores de los caracteres morfométricos de Ptilopora aff. bohemica se encuentran en la Tabla 4.

\section{Discusión}

El material estudiado se asemeja a Ptilopora bohemica fundamentalmente en la anchura de cámaras zoeciales, distancia entre centros de aberturas en hilera, anchura de la abertura y distancia entre centros de ramas laterales, conforme a los valores aportados por McKinney y Kř́ž (1986). Hay que tener en cuenta, no obstante, que estos autores no tratan separadamente las medidas de los caracteres zoeciales en ramas principales y laterales. Ptilopora aff. bohemica se diferencia de $P$. bohemica por la presencia de nodos en la quilla y de estrías longitudinales en el reverso de la lámina basal, ausentes en el material checo. Bigey (1972) cita la presencia de Ptilopora aff. bohemica en el Emsiense del Macizo Armoricano. Esta especie es muy similar a la nuestra en cuanto a la morfología zoarial; no obstante, las dimensiones de las cámaras son mayores y las de las aberturas zoeciales son más pequeñas que las de nuestros especímenes. Sin embargo, estas comparaciones no son absolutamente fiables dada la escasez de ejemplares disponibles por ambas partes. Bigey y Feist (1975) describen una especie, Ptilopora sp., del Emsiense superior de la Montaña Negra, a partir de ejemplares silicificados. Los caracteres medidos de estos ejemplares presentan en general mayores dimensiones que los de Ptilopora aff. bohemica.

Ptilopora bohemica fue definida en la localidad tipo de las Calizas de Koneprusy (Devónico Inferior, Bohemia) y revisada por McKinney y Kříž (op. cit.).

Filites Barrande, 1894

Especie tipo: Filites bohemicus Barrande in Pocta, 1894.

\section{Diagnosis}

Zoarios en forma de abanico poco organizados. Ramas dicotómicas con pinnas cortas, recurvadas hacia el reverso de la rama principal y fusionadas a ella. Ramas con reverso fuertemente espesado por esqueleto laminar. Zoecios inflados en forma de saco, con tubo distal ancho y formas trapezoidales o triangulares en sección tangencial profunda. Zoecios menos regulares en las pinnas. Pared axial en zig-zag. No hay quilla ni superestructura. (McKinney y Krríž, 1986).

\section{Filites sp. A}

Lám. II, figs. 6-10

\section{Descripción}

Zoario pinnado, constituído por una rama principal de la que salen sin periodicidad aparente ramas laterales

\section{Lámina II}

1 Polypora aff. inusitata McKinney y Kříž, 1986. Sección transversal mostrando las crestas en la lámina basal. PIC-29-2, x28.

2-5 Ptilopora aff. bohemica Prantl, 1928. 2: sección longitudinal de una rama principal cortando cámaras con sus tubos distales. PIC-28s-3, x26. 3: sección longitudinal de una rama principal mostrando en detalle los tubos distales de los zoecios. PIC-67-2, x86. 4: sección transversal de una rama principal con crestas bien definidas en la lámina basal y esqueleto laminar espeso. PIC-33-1, x86. 5: sección tangencial profundizando hacia la parte superior de la fotografía. Se aprecian nodos en la quilla, y la morfología de aberturas y cámaras zoeciales, PIC-28s-3, x26.

6-10 Filites sp. A. 6: sección transversal de dos zoarios afectados por silicificación parcial. En el de la derecha se distingue el tubo distal de una cámara. PIC-29s-1, x28. 7: sección tangencial de un zoario, mostrando las hileras de zoecios de la rama principal y las inserciones de las pinnas. PIC-28-4, x26. 8: sección transversal del mismo ejemplar cortando una cámara de la rama principal y los zoecios de una pinna (derecha), x79. 9: sección longitudinal del mismo ejemplar cortando las pinnas con sus zooecios, x26. 10: sección longitudinal del mismo ejemplar a lo largo de la rama principal, $\mathrm{x} 26$. 

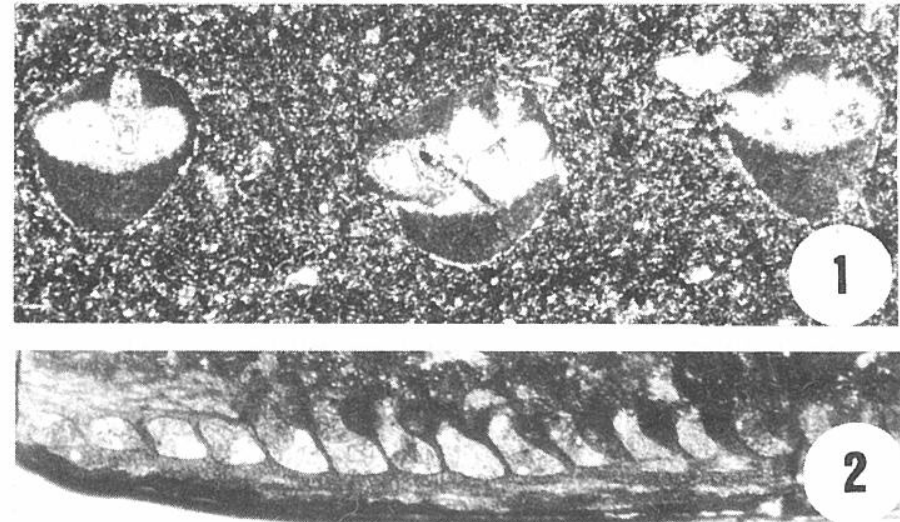

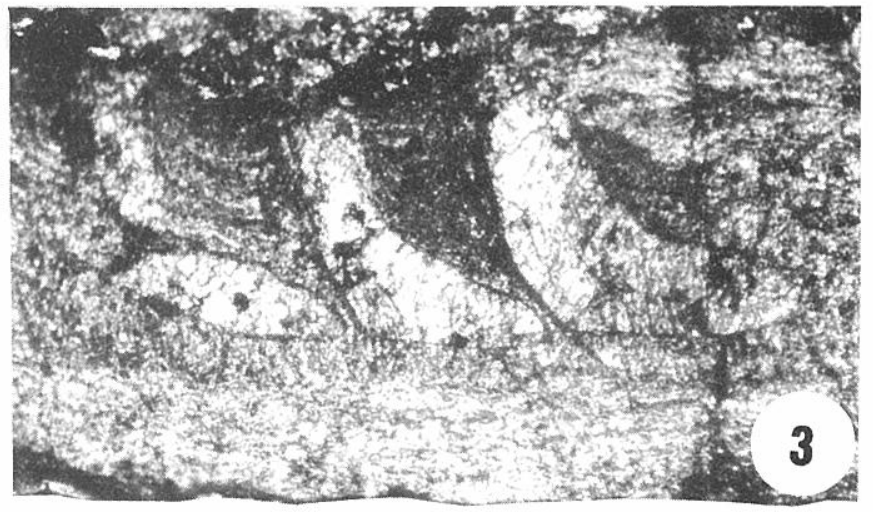

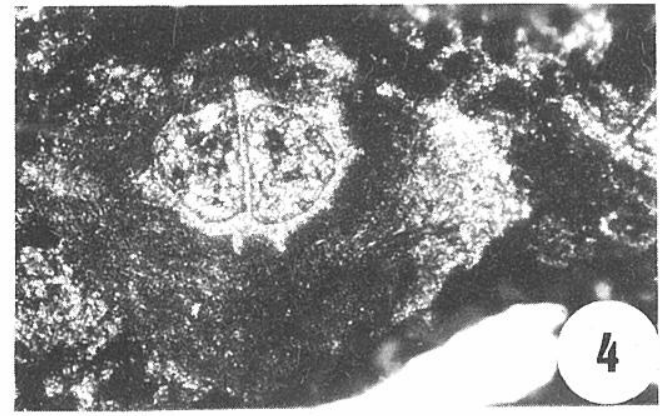
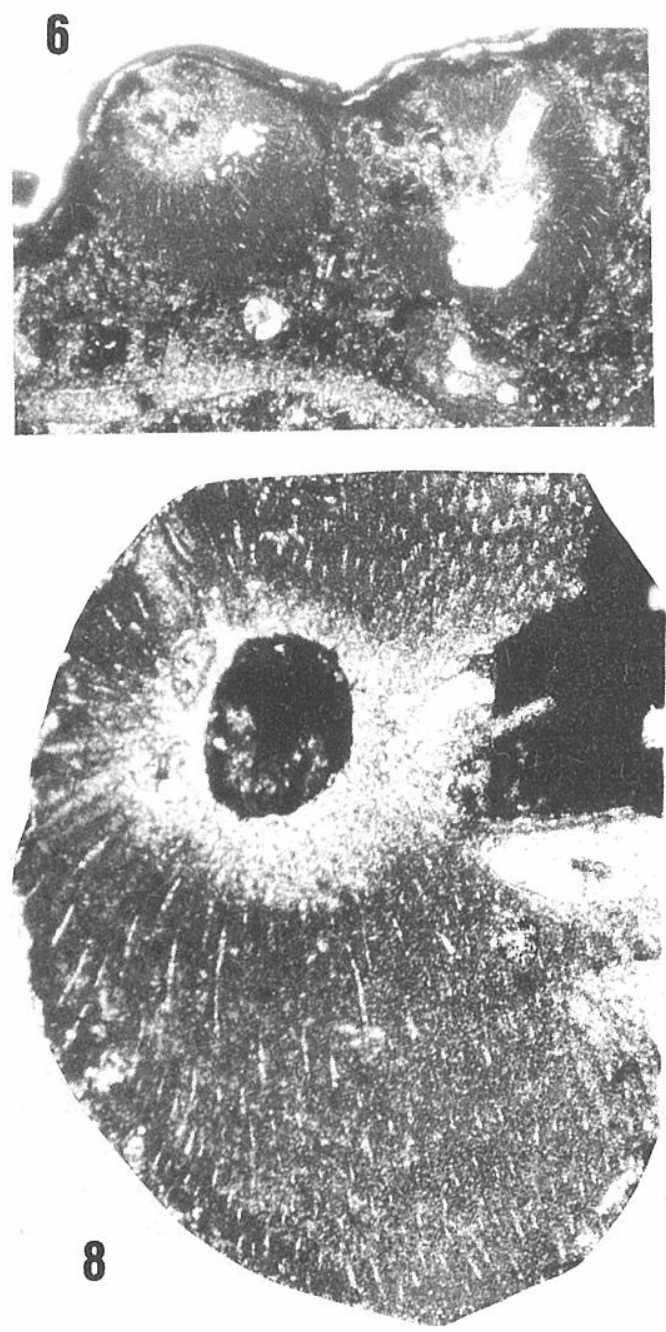
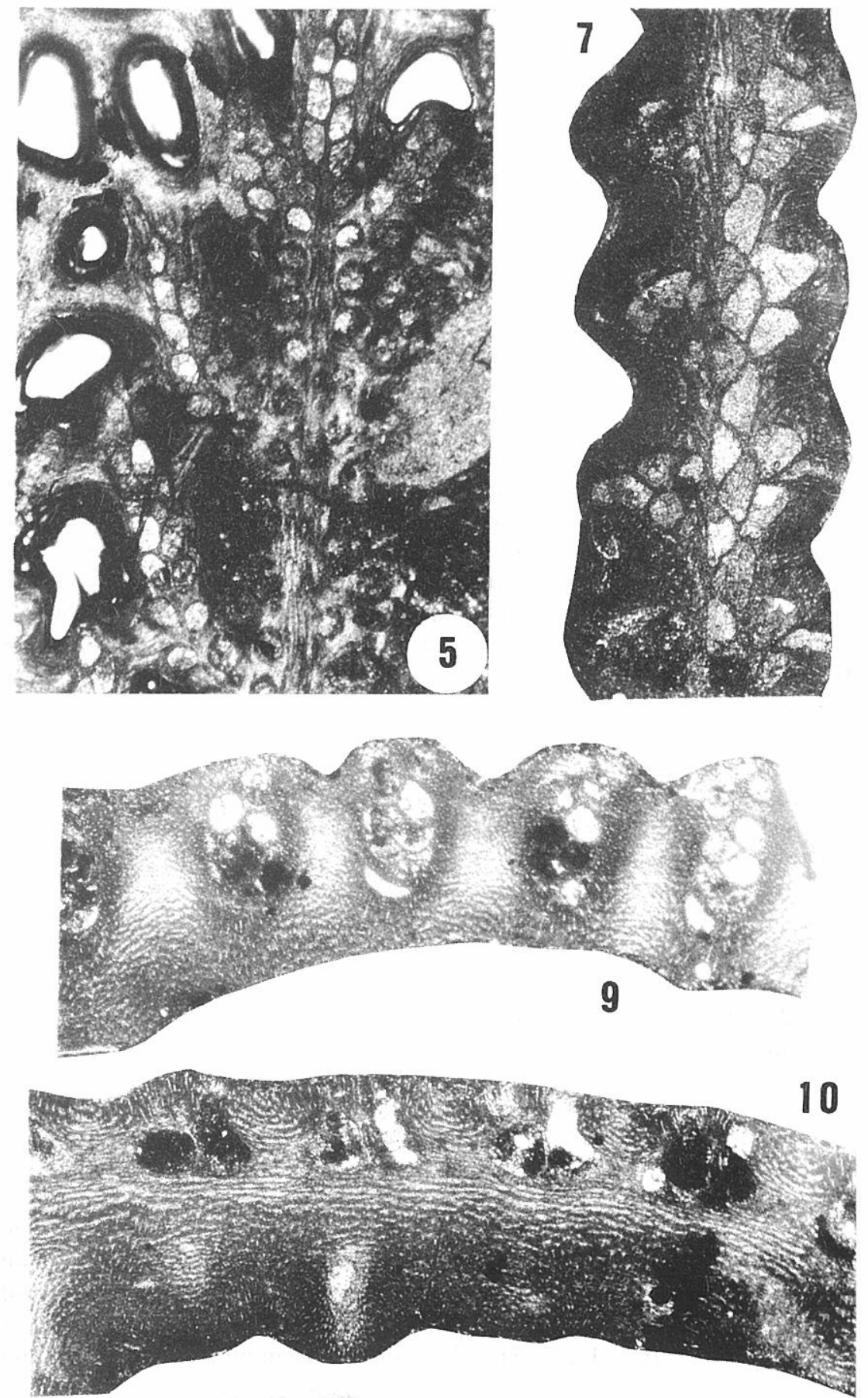

Revista Española de Paleontología, nº extr. Homenaje al Prof. J. Truyols, 1999. 


$\begin{array}{lcccc} & \text { media } & \text { desviación } & \text { rango } & \text { cf. varianza } \\ \text { ER } & 0,9417 & 0,1227 & 0,75-1,14 & 13,03 \\ \text { AR } & 1,0025 & 0,1310 & 0,8-1,30 & 13,06 \\ \text { *DAH } & 0,40 & - & - & - \\ \text { *AA } & 0,0960 & - & - & - \\ \text { MAC } & 0,1558 & 0,0101 & 0,145-0,18 & 6,50 \\ \text { LC } & 0,2960 & 0,0160 & 0,27-0,32 & 5,39 \\ \text { *ARC } & 79 & - & - & -\end{array}$

Tabla 5. Medidas de Filites sp. A. Las abreviaturas de los caracteres utilizados se explican en la Introducción. Las medias y rangos están expresados en mm, excepto el carácter ARC, en grados.

aproximadamente perpendiculares. Ramas principal y laterales de aspecto nodoso debido a la presencia de pinnas dispuestas de modo alternante y más o menos solapado. Pinnas con dos hileras de zoecios alternantes, que parten de la rama en su mismo plano y perpendiculares a ella y que se curvan hacia el reverso sin despegarse de la rama (Lám. II, figs. 7-9).

Ramas principal y laterales provistas de dos hileras de aberturas alternas, sin peristoma. Zoecios de las ramas dispuestos en dos hileras alternantes imbricadas, dando la apariencia de una sola hilera. Pared axial en zig-zag. Cámaras zoeciales con la pared lateral inflada; con forma triangular o de almendra en sección tangencial profunda y subcircular en secciones más superficiales (Lám. II, fig. 7). Tubo distal con diámetro ligeramente inferior a la anchura máxima de la cámara y profundidad aproximadamente mitad de la del zoecio. Los tubos distales divergen aproximadamente $20^{\circ}$ del plano axial de la rama, de manera que forman dos hileras, muy próximas, de aberturas. Los tubos distales salen al exterior casi perpendiculares a la superficie de la rama (Lám. II, figs. 6, 10).

Pinnas con dos hileras alternas de zoecios ligeramente imbricadas, dando dos hileras de aberturas redondeadas y sin peristoma, muy próximas entre sí, no separadas por quilla. En sección tangencial profunda y media, los zoecios tienen forma de saco, con la parte proximal adaptada a la forma del zoecio inmediatamente anterior y la parte distal fuertemente inflada. En secciones más someras, el perfil se redondea y estrecha ligeramente para dar lugar al tubo distal (Lám. II, figs. 7, 9). Esqueleto laminar muy espeso, especialmente en el reverso de las ramas, atravesado por multitud de estiletes de aproximadamente $0,007 \mathrm{~mm}$, que parten del esqueleto granular y alcanzan la superficie (Lám. II, fig. 8). Los valores de los caracteres morfométricos de Filites sp. A se encuentran en la Tabla 5.

\section{Discusión}

Filites sp. A no parece mostrar diferencias notables en los caracteres zoeciales con respecto a Filites bohemicus, la especie tipo. Sin embargo, hay diferencias en cuanto a la anchura de la rama, mayor en Filites sp. A, y el diámetro de los estiletes, ligeramente mayor en Filites bohemicus. Por esta razón no parece prudente en este momento asignar Filites sp. A a la especie tipo o crear una nueva especie, y se ha preferido dejarla en nomenclatura abierta en tanto no se preparen y analicen nuevas secciones.

\section{CONSIDERACIONES PALEOBIOGEOGRÁFICAS}

Bigey (1985) enmarca los briozoos devónicos de la Zona Cantábrica en la Magnafacies Eifeliana o Mixta de la Provincia Bohemo-Rhenana, en el Dominio del Viejo Mundo (Boucot y Gray, 1979). Para ello se apoya en los datos de Barrois (1882) y en estudios de braquiópodos que sitúan el dominio Astur-Leonés de la Zona Cantábrica en dicha magnafacies.

a) La mayor parte de los géneros estudiados en este trabajo poseen distribuciones geográficas y estratigráficas amplias, salvo Cyclopelta y Filites. Cyclopelta ha sido citado dentro de la Provincia Bohemo-Rhenana en el Eifel (donde se definió el género), en el Devónico Inferior de Bohemia y por primera vez en España, en la Cordillera Cantábrica, alcanzando una edad Eifeliense (Fig. 1).

b) Filites fue definido en el Devónico Inferior de la Cuenca de Praga y no se conoce en otras localidades; es por tanto la primera vez que se cita en España e incluso fuera de su localidad tipo, quizá debido a la escasez de estudios. Filites sp. A aparece en Asturias con una edad Emsiense superior, mientras que en Bohemia la especie tipo se ha citado sólo hasta el Zlichoviense. Por otra parte, Polypora cf. laevinodata, que aparece en niveles que posiblemente alcancen el Eifeliense inferior, es muy similar a Polypora laevinodata (Hall), del Eifeliense superior de Indiana.

c) Se ha identificado también el género Intrapora, un criptostomado bifoliado cuyas especies se han descrito casi exclusivamente en el Devónico Medio de Estados Unidos, salvo escasas citas de la antigua URSS. En Arnao, Intrapora aparece ya en el Emsiense. Estos datos parecen coincidir con los presentados por Soto (1979, 1982) para corales rugosos, Arbizu (1978) para trilobites y Racheboeuf (1981) para braquiópodos. En estos trabajos se menciona la existencia de comunicación ya en el Devónico Inferior entre el Dominio del E de Norte América y el Viejo Mundo.

\section{CONCLUSIONES}

1. La Formación Moniello posee una importante diversidad de briozoos, estando representados por cinco órdenes (Suárez Andrés, 1998, Introducción). Sólo dentro del Orden Fenestrida se han podido describir nueve especies pertenecientes a siete géneros, quedando otros géneros y especies aún por determinar debido a la escasez y a la conservación del material.

2. Destaca la distribución vertical especialmente corta de dos especies, Polypora cf. laevinodata y Filites sp. A. Sin embargo, no parece prudente su utilización como indicadores bioestratigráficos, dado que dichas distribuciones pueden estar condicionadas por los 
cambios de facies existentes, y aún no han sido comparadas en otras secciones equivalentes.

3. La fauna estudiada presenta similitudes con las de otras localidades de la Provincia Bohemo-Rhenana, en el Dominio del Viejo Mundo, especialmente con Bohemia. Sin embargo, algunos taxones son compartidos con el Dominio del E de Norte América, coincidiendo con la existencia de comunicación temprana entre ambos dominios, sugerida por varios autores.

\section{AGRADECIMIENTOS}

A los Dres. Soto, Bigey, Blake, Cuffey, Gilmour, Hageman, McKinney, Reguant, Snyder, Taylor y Tourneur, que con sus consejos y su bibliografía han hecho posible este trabajo. El trabajo es una contribución al Proyecto 421 del IGCP.

\section{BIBLIOGRAFÍA}

Arbizu, M. 1978. Trilobites Synphoriidae del Devónico de la Cordillera Cantábrica: Malladaiinae nov. subfam. Trabajos de Geología, 10, 37-65.

Barrois, Ch. 1882. Recherches sur les terrains anciens des Asturies et de la Galice. Mémoires Societé Géologique du Nord, 2 (1), 1-630.

Bigey, F. P. 1972. Présence de Ptilopora aff. bohemica (Bryozoaire Cryptostome) dans le Dévonien du Sud-Est du Massif armoricain. Bulletin de la Société d'Etudes Scientifiques de l'Anjou, 8, 15-22.

Bigey, F. P. 1985. Biogeography of Devonian Bryozoa. In: Bryozoa: Ordovician to Recent (Eds. C. Nielsen and G.P. Larwood), Olsen and Olsen, Fredensborg, 9-23.

Bigey, F. P. et Feist, R. 1975. Cryptostomata (Bryozoaires) des "Calcaires à Polypiers silicieux" (Dévonien Inferieur de la Montagne Noire, France Meridionale). Note preliminaire. In: Bryozoa 1974 (Ed. S. Pouyet). Documents du Laboratoire Géologique de la Faculté des Sciences de Lyon, H.S. 3(2), 257-271.

Boucot, A. J. and Gray, J. 1979. Epilogue: A Paleozoic Pangaea? In: Historical Biogeography, Plate Tectonics and the Changing Environment (Eds. J. Gray and A.J. Boucot). Oregon State University Press, Corvallis, 465482.

Hageman, S. J. 1991. Approaches to systematic and evolutionary studies of perplexing groups: an example using fenestrate Bryozoa. Journal of Paleontology, 65, 630-647.

Hall. J. 1881. Bryozoans of the Heldelberg and Hamilton groups. Transactions of the Albany Institute, 10. [Distributed in the form of abstracts separately paged].

McKinney, F. K. and Kř́iż, J. 1986. Lower Devonian Fenestrata (Bryozoa) of the Prague Basin, Barrandian Area, Bohemia, Czechoslovakia. Fieldiana Geology, N S, 15, 1-90.

Morozova, I. P. and Lisitsyn, D. V. 1996. Revision of the Genus Polypora McCoy, 1844. Paleontological Journal, 30, 530-541.

Nickles, J. M. and Bassler, R. S. 1900. A synopsis of American fossil Bryozoa including bibliography and synonymy. Bulletin of the United States Geological Survey, 173, $663 \mathrm{pp}$.

Prantl, F. 1928. Some Bryozoa from the Koneprusy Limestone (Bohemia). Bulletin international de l'Académie Chéque des Sciences, 29, 156-158.

Racheboeuf, P. R. 1981. Chonétaceés (Brachiopodes) Siluriens et Dévoniens du Sud-Ouest de l'Europe. Mémoires de la Societé géologique et minéralogique de Bretagne, 27, 1-294.

Soto, F. 1979. Considérations paléobiogéographiques sur les Streptelasmatina (Coelenterata, Rugosa) solitaires du Dévonien des Monts Cantabriques (NW de l'Espagne). Geobios, 12, 399-409.

Soto, F. 1982. Synaptophyllum (Rugosa) aus dem Unterdevon des Kantabrischen Gebirges (Colle, Prov. León). Neues Jahrbuch für Geologie und Paläontologie Abhandlungen, 163, 236-238.

Stratton, J. F. and Horowitz, A. S. 1977a. Variability in seven Devonian species of Polypora M'Coy. Bloomington, Indiana, 1-23.

Stratton, J. F. and Horowitz, A. S. 1977b. Polypora M'Coy from the Devonian of Southeastern Indiana. State of Indiana Department of Natural Resources, Geological Survey Bulletin, 56, 1-48.

Suaréz Andrés, J. L. 1997. Fenestrata (Bryozoa) de la Formación Moniello en el área de Arnao. Tesis de Licenciatura (inédita). Departamento de Geología, Universidad de Oviedo, 48 pp.

Suaréz Andrés, J. L. 1998. Briozoos Fenestrados de la Formación Moniello (Devónico) en el área de Arnao (Asturias, España). I: Fenestellidae. Revista Española de Paleontología, 13, 187-196. 